\title{
Documento per la ripresa della vita scolastica
}

Rita Librandi, Claudio Giovanardi e Francesco Sabatini

PUBBLICATO: 24 APRILE 2020

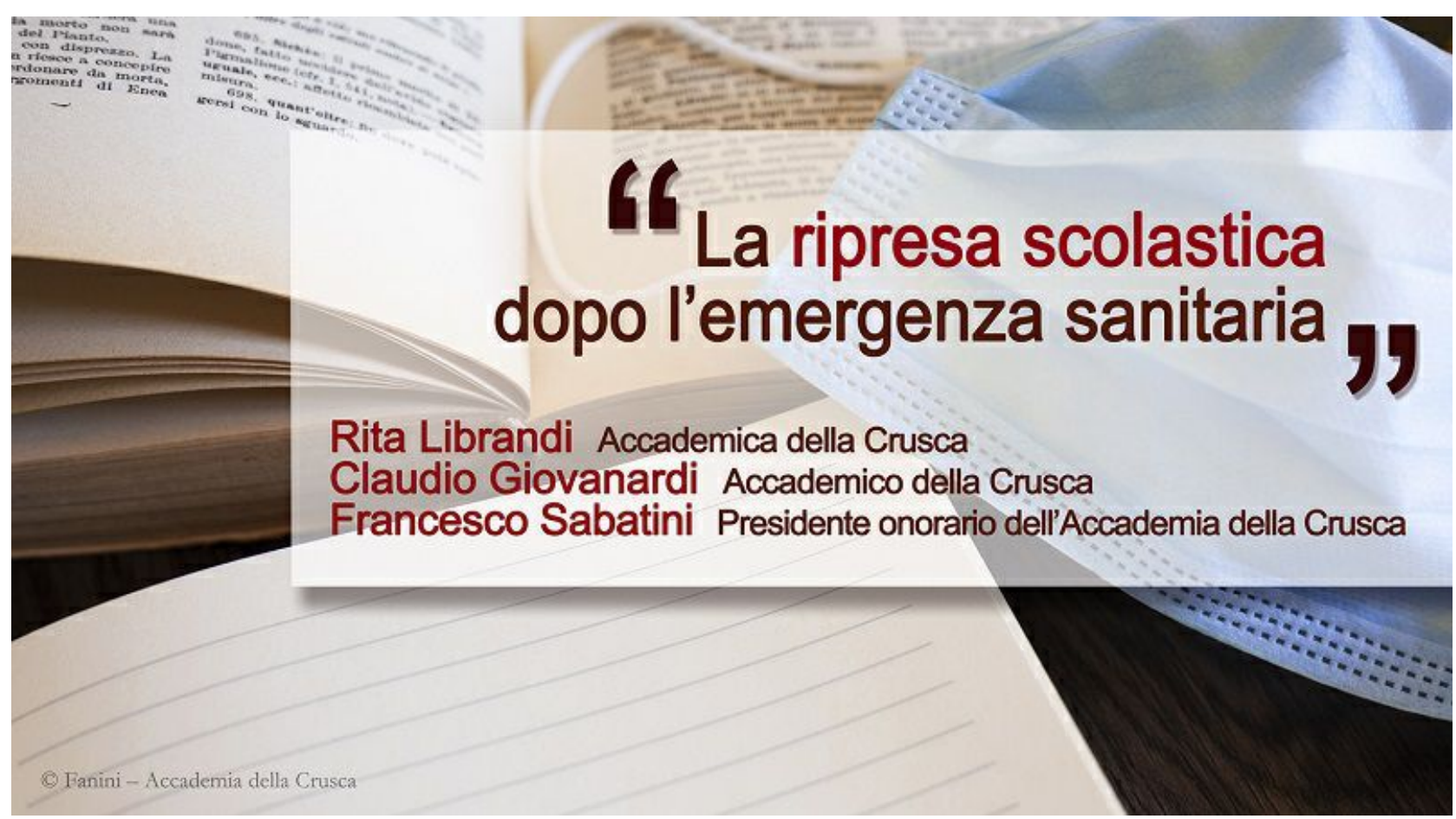

I

n una fase cosi delicata e difficile per il sistema scolastico italiano l'Accademia della Crusca, con la Sezione Crusca Scuola, e l'Associazione per la Storia della Lingua Italiana, Sezione Scuola, sentono il dovere di intervenire sui rischi di una cattiva interpretazione delle nuove modalità d'urgenza connesse soprattutto con l'insegnamento a distanza.

Il nostro corpo docente nel suo insieme ha reagito in modo esemplare davanti all'emergenza della pandemia, dimostrando piena consapevolezza del proprio ruolo e dell'alto valore che la formazione assume nella società: rimodulando procedure, forme di comunicazione, interazione con bambini e adolescenti, potenziando al massimo l'uso degli strumenti telematici o talvolta impadronendosene per la prima volta. È un merito che gli va prontamente riconosciuto. La risposta straordinaria, tuttavia, non deve far confondere tale capacità di intervenire in urgenza con la soluzione di un processo educativo che non può esaurirsi nella trasmissione di contenuti attraverso il web: la scuola è un'aula e non un video. Si tratta di un principio fondamentale tanto per la scuola quanto per l'università, che non vive con minore disagio l'impossibilità di tenere lezioni ed esami in presenza.

Gli stessi insegnanti, molto meglio di chiunque altro, stanno denunciando i limiti dell'insegnamento a distanza, limiti peraltro già da tempo sottolineati dagli esperti di pedagogia e didattica. I difetti riscontrati da più parti sono tanti e non è inutile ricordarli.

- L'insegnamento via web non consente di verificare con immediatezza la risposta degli studenti alla lezione e il loro grado di comprensione dei contenuti esposti.

- La distanza rende più difficile valutare la giusta distribuzione temporale delle fasi di insegnamento e apprendimento, anche per la ridotta interazione tra chi parla e chi ascolta. 
- Si annullano, o almeno si riducono in modo essenziale, la socializzazione e il lavoro di squadra, impedendo che la classe funzioni come modello di interazione virtuosa tra i ragazzi e tra generazioni diverse in un fecondo scambio e arricchimento reciproco.

- Si riduce la fisicità dell'insegnamento, che non riguarda solo la gestualità con cui l'insegnante accompagna le spiegazioni, sottolineandone i punti salienti o elevandone le emozioni, ma anche e soprattutto l'abilità manuale guidata fisicamente, che non può essere dimenticata nell'apprendimento della scrittura. Molti sono ormai gli studi che in tempi recenti hanno dimostrato quanto sia importante, per lo sviluppo delle capacità cognitive, conservare, nella scuola primaria, l'apprendimento della scrittura manuale, non disperdendola a favore di quella digitale.

I limiti della distanza non sono, però, soltanto di natura strettamente didattica. Un sistema di insegnamento, infatti, per il quale è indispensabile possedere strumentazioni adeguate, buone connessioni e stanze in cui potersi concentrare, discrimina vistosamente i più svantaggiati, né può servire una sia pur meritevole distribuzione di tablet alle famiglie più povere: senza genitori in grado di affiancare lo sforzo dei discenti, senza libri nelle case, senza spazi adeguati il problema non si risolve.

Gli aspetti negativi di una didattica a distanza non riguardano ovviamente l'uso sapiente delle tecnologie informatiche nell'istruzione, la possibilità di integrare l'insegnamento con le risorse del web, che hanno dato e continueranno a dare un contributo di grandissima efficacia.

Il nostro appello va a coloro che devono, appena possibile, garantire un ritorno migliorato all'attività educativa ordinaria, in analogia a quanto si cerca di fare nel settore sanitario. I fatti presenti confermano che la salute è il bene primario, ma confermano altresi che tutti gli aspetti della vita di ciascun Paese, compreso quello ora nominato, dipendono dagli investimenti nell'istruzione, nella ricerca, nel diritto allo studio, beni da assicurare alle generazioni crescenti, energie indispensabili anche da trattenere il più possibile nella terra di origine.

Gli sforzi encomiabili per far fronte alla pandemia sono stati tanti: pensiamone tanti anche per la riapertura delle scuole. Come si è riconosciuta inadeguata la forza numerica umana nel campo della sanità, per effetto delle restrizioni improprie e di spirito elitaristico, cosi si riconosca che l'affollamento nelle classi è stato un provvedimento ministeriale sconsiderato; si provveda con l'occasione a rendere accettabile il rapporto numerico discenti - docenti. E non si assecondi la convinzione - forse gradita in ambienti solo commercialmente interessati - che la scuola possa essere un video e non un'aula: sarebbe, non solo nella battaglia contro la Covid-rg, una sconfitta irreparabile.

\section{Cita come:}

Rita Librandi, Claudio Giovanardi e Francesco Sabatini, Documento per la ripresa della vita scolastica, "Italiano digitale", 2020, XIII, 2020/2 (aprile-giugno)

DOI: $10.35948 / 2532-9006 / 2020.3322$

Copyright 2020 Accademia della Crusca

Pubblicato con licenza creative commons CC BY-NC-ND 